\title{
Video Article \\ Murine Myocardial Infarction Model using Permanent Ligation of Left Anterior Descending Coronary Artery
}

\author{
Jérôme Lugrin ${ }^{1,2}$, Roumen Parapanov ${ }^{1,2}$, Thorsten Krueger $^{2}$, Lucas Liaudet $^{1}$ \\ ${ }^{1}$ Service of Adult Intensive Care Medicine, Department of Interdisciplinary Centers and Logistics, Lausanne University Hospital and Faculty of Biology and Medicine, \\ Lausanne University \\ ${ }^{2}$ Service of Thoracic Surgery, Department of Surgery and Anesthesiology Services, Lausanne University Hospital and Faculty of Biology and Medicine, Lausanne \\ University
}

Correspondence to: Lucas Liaudet at Lucas.Liaudet@chuv.ch

URL: https://www.jove.com/video/59591

DOI: doi:10.3791/59591

Keywords: Medicine, Issue 150, Myocardial, infarct, mouse, permanent, ischemia, LAD, coronary artery, ligation

Date Published: 8/16/2019

Citation: Lugrin, J., Parapanov, R., Krueger, T., Liaudet, L. Murine Myocardial Infarction Model using Permanent Ligation of Left Anterior Descending Coronary Artery. J. Vis. Exp. (150), e59591, doi:10.3791/59591 (2019).

\section{Abstract}

Myocardial infarction (MI) and acute coronary diseases are among the most prominent causes of death in population with western lifestyle. The murine models of MI with permanent ligation of left-anterior descending (LAD) coronary artery closely mimics MI in humans. Murine models benefit from the extensive genetic engineering available nowadays. Here we propose a reproducible murine surgical model of myocardial infarction by permanent LAD coronary ligation. Our technique comprises anesthesia with ketamine/xylazine that can be rapidly reversed by administration of an antagonist, intubation without tracheotomy for mechanical-assisted ventilation, ventilation with application of extrinsic positive end-expiratory pressure (PEEP) to avoid alveolar collapse, a thoracotomy method limiting to the minimum surgical lesions made to skeletal muscles, and lung inflation without thoracentesis. This method is sparsely invasive, reproducible and reduces post-surgery mortality and complications.

\section{Video Link}

The video component of this article can be found at https://www.jove.com/video/59591/

\section{Introduction}

Acute myocardial infarction (MI) is the most severe expression of ischemic heart diseases (IHD). IHD are the leading cause of morbidities and death worldwide, especially in western countries ${ }^{1}$. Consequently, it has an enormous economic impact on healthcare systems ${ }^{2}$. MI is characterized by the occlusion of a coronary artery by atherosclerotic plaque and the subsequent arrest of blood flow in large parts of the myocardium. Lack of oxygen supply in the myocardium leads to ischemic death of cardiomyocytes. This pathological condition triggers responses in the ventricular tissue that ultimately leads to deficiencies in ventricular functions, remodeling and heart failure ${ }^{3}$. MI is a complex pathophysiological condition that involves multiple and intricate biological processes comprising regulated cell death, response to oxidative stress, inflammation, wound healing, fibrosis and ventricular remodeling. Some of these biological responses are modeled as individual processes in vitro like necrosis-induced release of damage-associated molecular patterns and associated inflammatory responses ${ }^{4}$. These simplified models are essential to understanding MI. However only an in vivo model can provide a realistic image of the biological processes complexity engaged in response to MI.

Even though models of $\mathrm{MI}$ in larger animals like swine may more closely relate to human pathophysiology of MI, the power of the murine models resides in the possibilities offered by genetic engineering that is more advanced than in any other mammal species. Other non-negligible aspects are the relative low cost and the simplicity of the surgical setup.

It is worth to mention that models of ischemia-reperfusion of the myocardium can exhibit different outcomes than permanent MI models. Biological processes like the type of cell death engaged, quality/amplitude or kinetics of inflammatory and wound healing responses in the myocardial tissue might vary according to the model ${ }^{5,6,7}$. However, this protocol of permanent coronary occlusion can easily be adapted to obtain an ischemia-reperfusion model.

This method is relevant for studies related to the physiopathology of MI without reperfusion and allows monitoring of pathological processes occurring from coronary occlusion (minutes) to late stage heart failure (weeks) at the local heart tissue and systemic levels.

\section{Protocol}

Animal experiments described in this protocol were reviewed and approved by Animal Ethics Committee of Canton of Vaud. 
NOTE: For these experiments, we used male C57BI/6J mice weighing between $25 \mathrm{~g}$ and $30 \mathrm{~g}$ and an age of 8-12 weeks. Mice were fed chow pellets and water ad libitum and bred under conventional conditions. Surgical equipment was previously sterilized. The experimenter should wear sterile surgical gloves and a surgical mask to limit contamination and post-operative infections.

\section{Anesthesia and tracheal cannulation.}

1. Weigh the mouse to determine the dosage of anesthetic drugs, post-operative analgesic medication and tidal volume of the ventilator. Prewarm the heating pad at $37^{\circ} \mathrm{C}$. The surgical setup is depicted in Figure 1.

2. Inject mouse intraperitoneally with a mix of ketamine and xylazine at a dose of $80 \mathrm{mg} / \mathrm{kg}$ and $10 \mathrm{mg} / \mathrm{kg}$ respectively.

3. Quickly shave the mouse fur on the throat and the left side of the rib cage using an electric razor.

4. Check depth of anesthesia by pinching tail and/or hind feet and settle the animal in a supine position on the heating pad. Place a small gauze compress under the head of the animal to avoid overheating of the eyes. Apply ocular gel to avoid eye dryness.

5. Secure the four limbs with adhesive tape on the surface of the heating pad. Pass a loop of 5-0 silk suture under the upper incisors and stick the extremity of the loop with adhesive tape onto the heating pad. This will keep the mouth of the animal open and facilitate cannulation.

6. Apply hair removal cream on the pre-shaved areas and gently massage with a cotton swab for 1 min. Wipe the excess of fur and cream with a gauze. Use drops of $0.9 \%$ saline solution and gauze to clean the incision areas. Apply pieces of sterile gauze to the shaved throat and thorax and soak them in iodopovidone.

NOTE: We recommend application of a local anesthetic drug (lidocaine or bupivacain) to incisions sites.

7. Set the ventilator at a tidal volume of $7 \mathrm{~mL} / \mathrm{kg}$ and ventilation rate of 140 strokes $/ \mathrm{min}$. NOTE: From now on work under a microsurgery stereomicroscope.

8. Hold the skin on the center of the throat and perform an incision of $0.5 \mathrm{~cm}$ following a caudal/cephalic line using small scissors. Separate the lobes of salivary gland, then gently separate fascia of sternohyoid muscle with curved dissecting forceps until larynx and trachea are visible. Secure edges of the opening with retractors attached to elastic bands.

NOTE: Do this step without incision of the muscles. A trained operator will be able to intubate the animal via oral cavity without visualization of the trachea making this step optional.

9. Hold gently the tongue sideways. With forceps, insert the blunted inner needle of a $16 \mathrm{G}$ cannula into the trachea. Visualize correct insertion into the trachea through the throat incision.

10. Connect the cannula to the ventilator and ensure correct ventilation by placing the exhaust tubing into water. The presence of bubbles indicates correct intubation.

NOTE: In order to keep tissues wet during operation place sterile gauze soaked with $0.9 \%$ saline solution and iodopovidone on the throat incision. Control moisture during the procedure.

\section{Ligation of LAD coronary artery}

1. Release left anterior paw from duct tape and carefully move the mouse to right side decubitus position. Secure the left anterior limb once animal is in the correct position.

2. Identify the line between left pectoralis minor and major muscles and make an oblique skin incision on $1 \mathrm{~cm}$ with scissors following the line. With dissecting blunt micro scissors, separate fascia of pectoralis muscles without incision. Maintain pectoralis muscles separated with retractors attached to elastic bands.

3. Set the ventilator with a positive end-expiratory pressure (PEEP) of $3 \mathrm{~cm} \mathrm{H}_{2} \mathrm{O}$.

4. Open the chest cavity by using blunt forceps at the $3^{\text {rd }}$ intercostal space between $3^{\text {rd }}$ and $4^{\text {th }}$ ribs. Avoid touching internal thoracic artery as there is danger of bleeding. Do not touch heart or lung. Apply two retractors into the ribcage, one on each rib (Figure 2A).

5. With a curved fine forceps, carefully remove the pericardium and pull it apart without harming the heart and lungs.

6. Locate left anterior descending (LAD) coronary artery. LAD artery appears as a superficial bright red line running from the edge of the left auricle toward the apex.

7. Use a needle holder to pass a 7-0 silk suture under the LAD 2 to $3 \mathrm{~mm}$ below left atria. Pull the silk slowly to avoid a tearing of heart tissue. Tie the ligature with three knots. The lower left part of the left ventricle will instantly turn pale upon ligation (Figure 2B-E). NOTE: It is important to not go too deep into the ventricular cavity or to stay too superficial. For sham-operated animals, pull the suture silk under the LAD and remove it slowly avoiding tissue tearing.

8. Release the rib retractors, hold the $3^{\text {rd }}$ rib with forceps and make two passes with a 6-0 silk suture under the $3^{\text {rd }}$ and $4^{\text {th }}$ ribs. CAUTION: Do not the perforate heart or lung. Do not tighten knots yet.

9. Put three drops of $37^{\circ} \mathrm{C} 0.9 \%$ saline solution onto the opening and shut the expiration exhaust tube for 2 or 3 respiratory cycles to properly inflate lungs. Tighten the suture and secure with two throws.

10. Release retractors holding muscles and help them retrieve their correct place.

11. Close thoracic skin with two stitches of 5-0 suture silk and secure with two throws. Close throat skin with one stitch of 5-0 suture silk and secure with two throws.

\section{Post-operative procedures and follow-up.}

1. Remove adhesive tape bands from limbs. Put a compress on the heating pad on the right side of the animal. NOTE: The overall procedure from anesthesia to this point should not take longer than $40-45 \mathrm{~min}$. Optionally inject IP $0.2 \mathrm{~mL}$ of atipamezole at a concentration of $0.1 \mathrm{mg} / \mathrm{mL}$ to speed up the waking up process.

2. Intraperitoneally inject $0.3 \mathrm{~mL}$ of $5 \%$ glucose solution pre-warmed at $37^{\circ} \mathrm{C}$

3. Carefully turn the animal on ventral decubitus onto the compress pad.

4. Stop ventilator; if the mouse spontaneously breathes, cautiously remove cannula.

5. Inject subcutaneous (SC) $0.1 \mathrm{mg} / \mathrm{kg}$ buprenorphine and put mice in a pre-warmed cage heated at $30{ }^{\circ} \mathrm{C}$ and ventilated with a $100 \% \mathrm{O}_{2}$ for a minimum of $1 \mathrm{~h}$. Monitor mice for any life-threatening condition such as excessive dyspnea or hemorrhage. 
6. During the two first days following surgery, monitor mouse twice daily. Inject SC $0.1 \mathrm{mg} / \mathrm{kg}$ buprenorphine twice daily. Intraperitoneally inject $0.3 \mathrm{~mL} 0$ of $5 \%$ glucose solution twice daily. Provide mice with soft diet and water ad libitum. Warm up the animal if necessary. NOTE: In addition to opioids, animals should be provided with nonsteroidal anti-inflammatory drugs mixed in diet or diluted in drinking water.

7. From day three, inject SC $0.1 \mathrm{mg} / \mathrm{kg}$ buprenorphine twice daily if the animal exhibits any unusual signs concerning general appearance, respiration or behavior. Intraperitoneally inject $0.3 \mathrm{~mL}$ of $5 \%$ glucose solution twice daily if the animal is still losing weight. Warm the animal if necessary.

NOTE: Strictly apply predefined interruption criteria when necessary to avoid excessive suffering. Usually mice lose weight up to day 3 and 4 and then gain weight. After seven days, mice usually retrieve pre-operation weight.

\section{Representative Results}

Mice were euthanized seven days after surgery. Animals were anesthetized with $80 \mathrm{mg} / \mathrm{kg}$ ketamine and $10 \mathrm{mg} / \mathrm{kg}$ xylazine. Under anesthesia, blood was drawn from vena cava and heart was sampled. Atria were removed, myocardium were washed in ice-cold PBS. For measurements of ischemic areas, hearts were frozen at $-20^{\circ} \mathrm{C}$ for $40 \mathrm{~min}$, then sliced and stained for 20 min at $37^{\circ} \mathrm{C}$ in PBS containing $2 \%$ triphenyltetrazolium chloride (TTC). Heart slices were fixed overnight in $4 \%$ buffered paraformaldehyde solution at room temperature. Ischemic areas remained unstained whereas live tissue was stained in red due to the presence of dehydrogenases. Ischemic areas were calculated as percentage of white area of the left ventricle (LV) with an imaging software (Figure 3A, B). For biochemical and molecular biology analyses, hearts were frozen in liquid nitrogen. After grinding hearts on liquid nitrogen the organ powder was used for protein and mRNA extraction. The extent of fibrosis in the myocardial tissue of infarcted hearts was assessed by western blot analysis of alpha smooth-muscle actin (aSMA) and SMAD2 phosphorylation, which are respectively major read-outs of myofibroblasts and of TGF $\beta$ signaling activation (Figure 3C). mRNA expression of Tgfb, and downstream targets Ctgf, Postn and I/11 are all indicators of myocardial fibrosis. This was shown by real-time polymerase chain reaction (PCR) analysis (Figure 3D).

Pro-inflammatory signaling pathways and expression of pro-inflammatory genes were typically found activated within the first week following myocardial infarction. Phosphorylation of NF-KB p65 transcription factor is a hallmark of inflammation and was observed in whole myocardium extracts of the MI mice (Figure 3E). mRNA expression of pro-inflammatory genes $/ / 1 \mathrm{~b}, / / 6$ and $\mathrm{Cxc} / 10$ (Figure 3F) and monocytes/macrophages markers Cd14 and Mertk were analyzed by real-time PCR (Figure 3G). Note that there was a variability in the extent of NF-KB p65 and SMAD2 phosphorylation (Figure 3C,E, lanes 4-7). This variability depends largely on the size of the infarct.
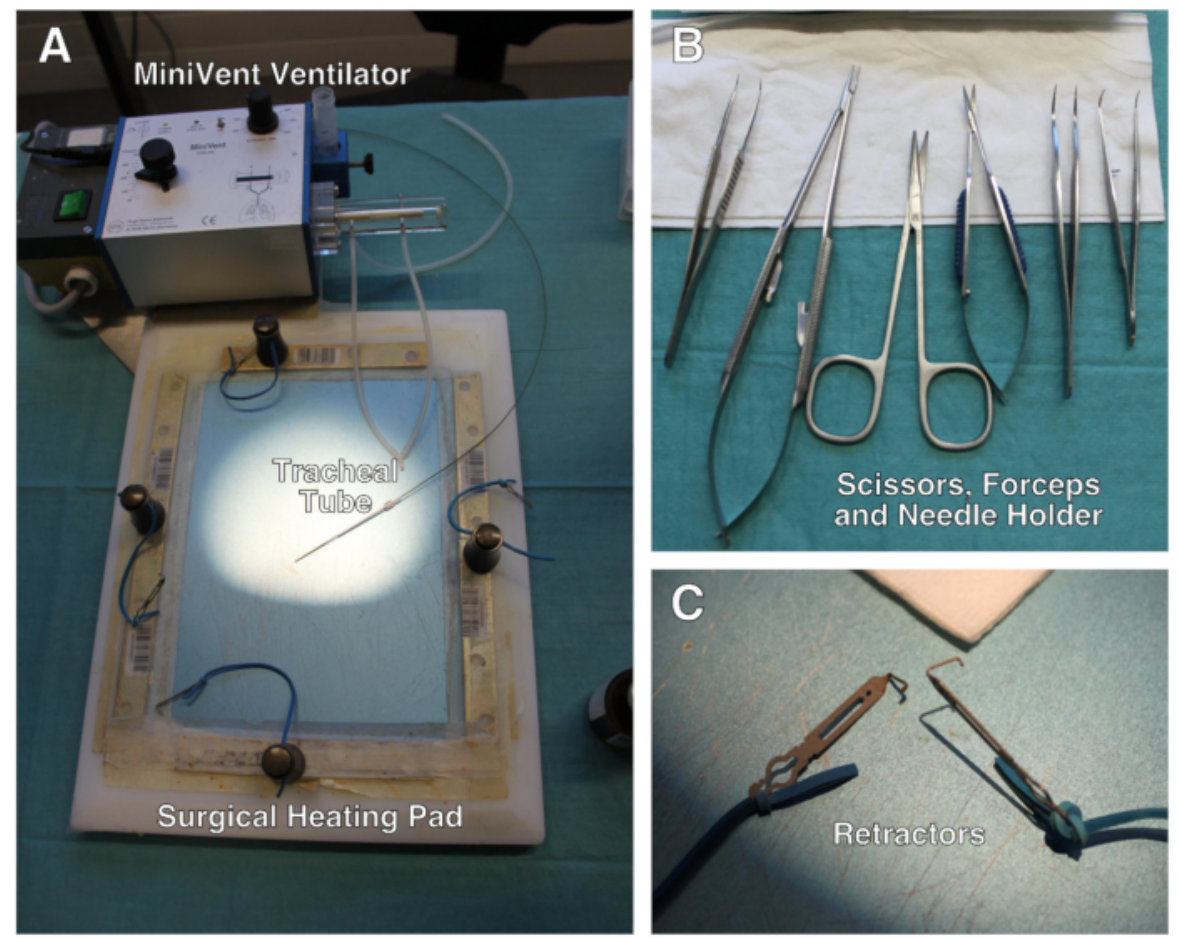

Figure 1: Description of the surgical setup. (A) Surgical setup comprises a modified heating pad, a ventilator and retractors attached to elastic bands. (B) Set of scissors, forceps and needle holder used during the surgery. (C) Close-up of the mini-retractors. Not shown: surgical stereo microscope. Please click here to view a larger version of this figure. 

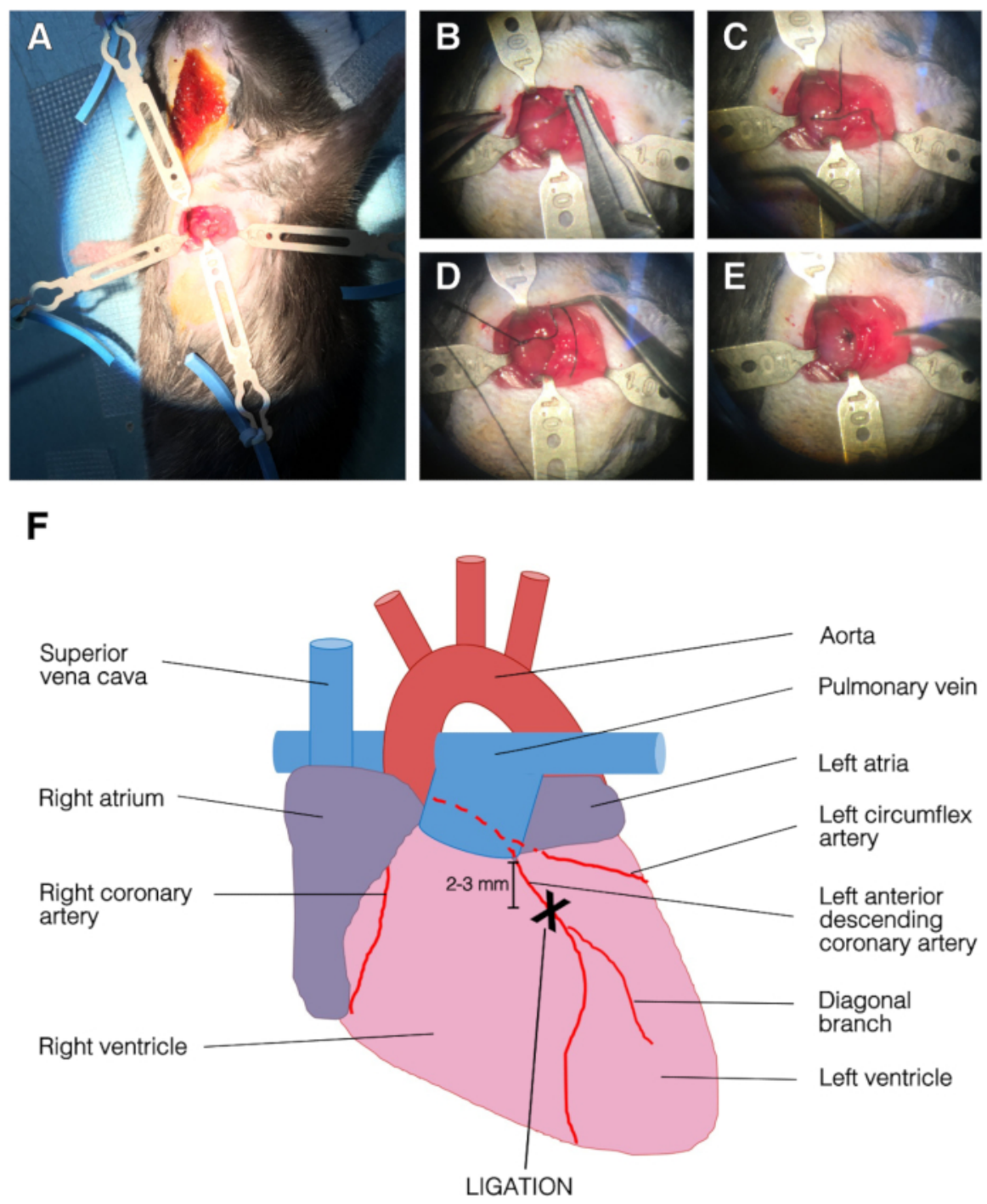

Figure 2: Representative images of the surgery and LAD ligation. (A) Opened chest with retractors. The left ventricle was apparent. Top, left and bottom retractors held the ribcage and right retractor held the pectoralis muscle. (B) The needle was passed under the LAD. (C) Suture silk was passed under the $L A D$, into the left ventricle. (D) Single stitch on the $L A D$. (E) End of the ligation procedure, the suture was secured with three knots. (F) Representation of an anterior view of the heart. The position of LAD ligation was 2-3 mm below left atria and above diagonal branch of the LAD. Please click here to view a larger version of this figure. 
A

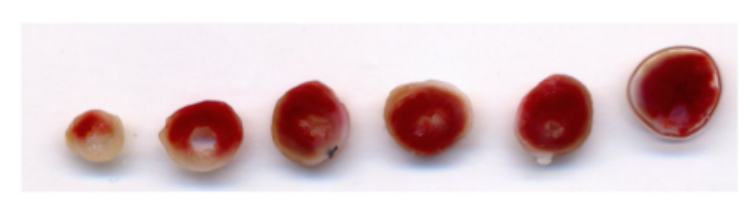

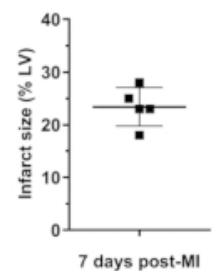

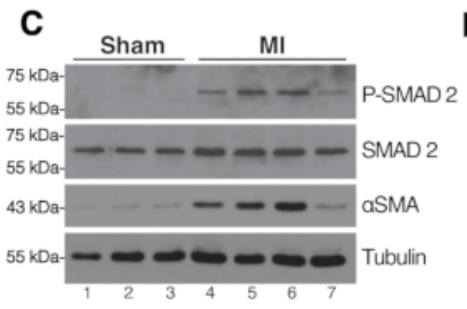

D
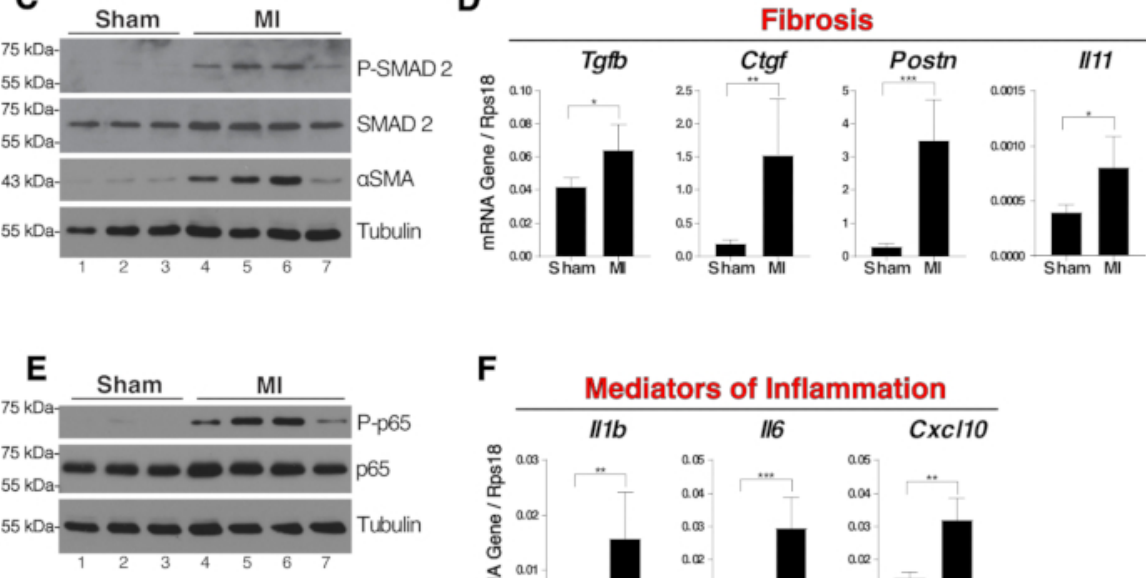

$\mathbf{F}$
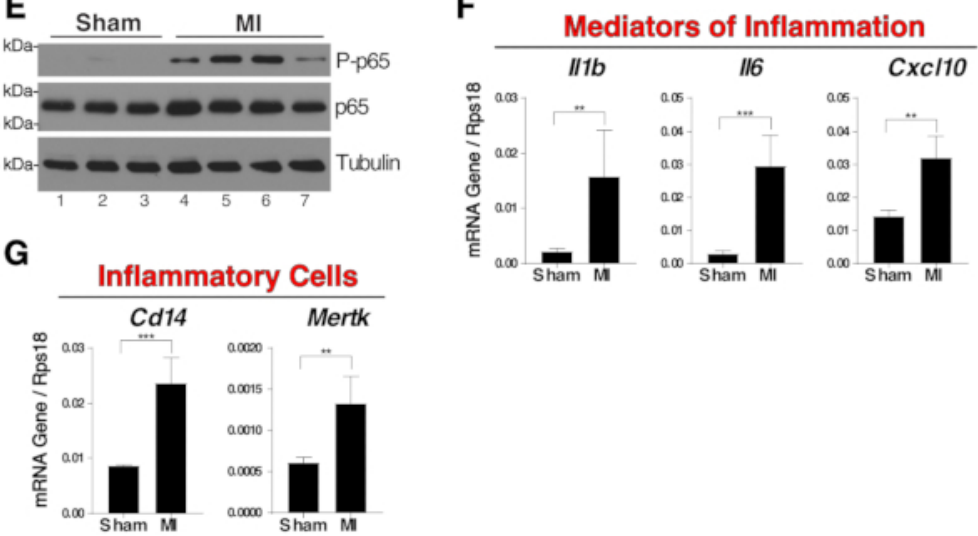

Figure 3: Fibrosis and inflammation in whole myocardium extracts seven days post-surgery. (A) Representative images of TTC staining of a sliced infarcted heart seven days post-surgery. Pale ischemic areas remained unstained and white whereas live tissue was stained red. The ligation was visible on the third slice from the left. (B) The size of the ischemic areas of five infarcted hearts were measured using TTC staining technique. Results were the percentage of white area of the left ventricle (LV). (C) Western blot analysis of SMAD2 phosphorylation and alphaSMA expression in whole myocardium as indicators of fibrosis. (D) mRNA expression of Tgfb, Ctgf, Posth and II11 in whole myocardium extracts. (E) Western blot of NF-KB p65 phosphorylation in whole myocardium extracts. (F) mRNA expression of pro-inflammatory genes $/ / 1 b$, $/ / 6$ and Cxcl10 in whole myocardium extracts. (G) mRNA expression of Cd14 and Mertk as indicators of the presence in the myocardium of monocytes/ macrophages and phagocytic macrophages respectively. $\mathrm{N}=3$ in sham and $\mathrm{N}=4$ in Ml group. For mRNA expression analysis, expression was relative to the endogenous control Rps18 and group comparisons were unpaired Student's T-tests, ${ }^{*} p \leq 0.05$, ${ }^{* *} p \leq 0.01$, ${ }^{* * *} p \leq 0.001$. In panels $\mathrm{B}, \mathrm{D}, \mathrm{F}$ and $\mathrm{G}$ error bars represent standard deviations. Please click here to view a larger version of this figure.

\section{Discussion}

The first critical step of this procedure is certainly intubation. We use the blunted inner needle of a $16 \mathrm{G}$ catheter as a tracheal tube. We do not recommend using this setup with mice that weight less than $22 \mathrm{~g}$. With this setup, it may be difficult to intubate mice properly with smaller bodyweight without damaging the trachea. Another critical point is to limit incisions made to the muscle while exposing the trachea and ribcage. Reducing tissue damage is of major importance, especially when studying inflammatory processes subsequent to MI. That is why we prefer gentle spreading of muscle and ribs with forceps and retractors ${ }^{8,9}$. We do not use electric cauterizer to control bleeding ${ }^{10}$. This may cause iatrogenic burns and favor infections. Both trauma and infections may bias inflammatory read-outs. Application of an extrinsic PEEP of $3 \mathrm{~cm} \mathrm{H}_{2} \mathrm{O}$ by plunging the ventilation exhaust into a water tube limits end-expiratory alveolar collapse during thoracotomy. Localization of LAD is another critical step and one should keep in mind that the anatomy of the coronary arteries may vary depending on the strain and the genotype of the mouse $^{11}$. It requires some experience to visualize the LAD, however placing the suture directly $2-3 \mathrm{~mm}$ below the left atria as described in the procedure shall allow correct positioning of the ligation. Instant discoloration of large portions of the left ventricle under the suture confirm the accuracy. Finally, artificially applying auto-PEEP by blocking ventilation exhaust for 2-3 respiratory cycles during chest closure allows a transient hyperinflation of the lung that will help chase the air from thoracic cavity ${ }^{12}$. We purposely do not perform a thoracentesis as shown in ${ }^{9,10}$. This way, we limit the risk of lung and heart injuries and avoid excessive tissue damage or perforation.

Myocardial ischemia-reperfusion (I/R) is a related surgical model that mimics the restoration of coronary blood flow that is done to $\mathrm{MI}$ patients in clinics. During the $\mathrm{I} / \mathrm{R}$ model a transient occlusion of the coronary artery is done by tightening a piece of tubing onto the LAD for a duration of 20 to $45 \mathrm{~min}^{8,13}$. Then the occlusion is released to allow reperfusion of the myocardium for the desired duration. This simple modification 
applied to our protocol can easily turn it into an I/R model $\left.\right|^{4,8,14,15}$. The infarction can be confirmed by a blood test for cardiac troponin $\mathrm{T}^{8,10}$ or by echocardiography ${ }^{15}$.

MI differs from I/R model because reperfusion by itself induces an injury. MI induces more tissue necrosis and apoptosis is more pronounced in reperfused myocardium ${ }^{5}$. Kinetics of inflammatory cells infiltration is also different between in MI and IR with a delayed myocardial infiltration of immune cells in $\mathrm{Ml}^{7}$. The size and the position of the infarcted area will also differ between permanent ligation and I/R models ${ }^{15}$. Keeping this in mind, one must be cautious to choose a relevant model since I/R and permanent $\mathrm{MI}$ models are not equivalent. Another murine model of myocardial infarction is the cryoinfarction model. Application of a cryogenic probe on the LV anterior wall induces the freezing of ventricular tissue and blood flow arrest in the LAD artery. This technique however differs from $\mathrm{MI}$ and I/R techniques regarding timing and amplitude of remodeling and inflammatory responses ${ }^{16,17}$.

Variability is a limitation as for any surgical procedure. This variability relies on biological differences. A good example is the variation in coronary arterial arrangement in mice ${ }^{11}$. It also relies on experimenter skills. It is worthwhile mentioning that adequate training of the experimenters is mandatory in order to reach stable outcomes with this model. A well-trained experimenter can easily produce infarct sizes that are reproducible (Figure 3A-B). The mortality of the model depends on the position of the LAD, duration of the experiments (days, weeks), mouse strain and genotypes. The types of anesthetic and analgesic drugs may also affect the outcome of the experiments with putative cardioprotective or cardiodepressant effects. In our hands, this model has a global mortality rate of $25-30 \%$. This mortality rate comprises spontaneous deaths and sacrifices before the end of the experiment, regardless strains and experiment duration. Most of the deaths or sacrifice are between the second and fourth days post-surgery. Applying a strict pain management and follow up of the animals can reduce mortality.

Here we present representative results of infarct size analyzed using TTC staining and expression of protein and genes involved in inflammatory or fibrotic processes in LV by western blot and real-time PCR respectively (Figure 3C-G). It is also possible to measure many of these parameters by enzyme-linked immunosorbent assay (ELISA) or enzymatic assays. Of course, in accordance with hypothesis that needs to be tested, this method can be followed by any functional analysis by ultrasound, MRI or intraventricular catheter measurement of pressure and volume. It is also possible to extract heart and further investigate cardiac cell biology on isolated cells. Overall, the MI model with permanent ligation of the LAD coronary artery is particularly useful to evaluate inflammatory and fibrotic processes, wound healing and changes in cardiac function subsequent to myocardial infarction.

\section{Disclosures}

The authors have nothing to disclose.

\section{Acknowledgments}

This model was developed with the support of the Swiss National Science Foundation (Grants 310030_162629 to LL) and departmental funds from the Services of Thoracic Surgery and Intensive Care Medicine of the Lausanne University Hospital. JL is recipient of a grant from the Emma Muschamp Foundation. We acknowledge the crucial support of veterinarians and animal facility staff of the Faculty of Biology and Medicine of the Lausanne University. We thank Dr. Giuseppina Milano from the Service of Cardiac Surgery of Lausanne University Hospital and Dr. Alexandre Sarre from Cardiovascular Assessment Facilty of Lausanne University for their technical hints.

\section{References}

1. GBD 2016 Causes of Death Collaborators. Global, regional, and national age-sex specific mortality for 264 causes of death, 1980-2016: a systematic analysis for the Global Burden of Disease Study 2016. Lancet. 390 (10100), 1151-1210, (2017).

2. Reed, G. W., Rossi, J. E., Cannon, C. P. Acute myocardial infarction. Lancet. 389 (10065), 197-210, (2017).

3. Frangogiannis, N. G. The inflammatory response in myocardial injury, repair, and remodelling. Nature Reviews Cardiology. 11 (5), 255-265, (2014).

4. Lugrin, J.et al. Cutting edge: IL-1alpha is a crucial danger signal triggering acute myocardial inflammation during myocardial infarction. Journal of Immunology. 194 (2), 499-503, (2015).

5. Hashmi, S., Al-Salam, S. Acute myocardial infarction and myocardial ischemia-reperfusion injury: a comparison. International Journal of Clinical and Experimental Pathology. 8 (8), 8786-8796, (2015).

6. van Zuylen, V. L.et al. Myocardial infarction models in NOD/Scid mice for cell therapy research: permanent ischemia vs ischemia-reperfusion. Springerplus. 4 336, (2015).

7. Yan, X.et al. Temporal dynamics of cardiac immune cell accumulation following acute myocardial infarction. Journal of Molecular and Cellular Cardiology. 62 24-35, (2013).

8. Xu, Z., Alloush, J., Beck, E., Weisleder, N. A murine model of myocardial ischemia-reperfusion injury through ligation of the left anterior descending artery. Journal of Visualized Experiments. 10.3791/51329 (86), (2014).

9. Reichert, K.et al. Murine Left Anterior Descending (LAD) Coronary Artery Ligation: An Improved and Simplified Model for Myocardial Infarction. Journal of Visualized Experiments. (122), (2017).

10. Kolk, M. V.et al. LAD-ligation: a murine model of myocardial infarction. Journal of Visualized Experiments. (32), (2009).

11. Fernandez, B.et al. The coronary arteries of the C57BL/6 mouse strains: implications for comparison with mutant models. Journal of Anatomy. 212 (1), 12-18, (2008).

12. Muthuramu, I., Lox, M., Jacobs, F., De Geest, B. Permanent ligation of the left anterior descending coronary artery in mice: a model of postmyocardial infarction remodelling and heart failure. Journal of Visualized Experiments. (94), (2014).

13. Xu, Z., McElhanon, K. E., Beck, E. X., Weisleder, N. A Murine Model of Myocardial Ischemia-Reperfusion Injury. Methods in Molecular Biology. 1717 145-153, (2018). 
14. Parapanov, R.et al. Toll-like receptor 5 deficiency exacerbates cardiac injury and inflammation induced by myocardial ischaemia-reperfusion in the mouse. Clinical Science. 129 (2), 187-198, (2015).

15. Curaj, A., Simsekyilmaz, S., Staudt, M., Liehn, E. Minimal invasive surgical procedure of inducing myocardial infarction in mice. Journal of Visualized Experiments. 10.3791/52197 (99), e52197, (2015).

16. van den Bos, E. J., Mees, B. M., de Waard, M. C., de Crom, R., Duncker, D. J. A novel model of cryoinjury-induced myocardial infarction in the mouse: a comparison with coronary artery ligation. American Journal of Physiology-Heart and Circulatory Physiology. 289 (3), H1291-1300, (2005).

17. Duerr, G. D.et al. Comparison of myocardial remodeling between cryoinfarction and reperfused infarction in mice. Journal of Biomedicine and Biotechnology. 2011 961298, (2011). 